\title{
Searching for parity violation with the LIGO-Virgo-KAGRA network
}

\author{
Katarina Martinovic $\odot,{ }^{1}$ Charles Badger $\odot,{ }^{1}$ Mairi Sakellariadou $\odot,{ }^{1,2}$ and Vuk Mandic ${ }^{3}$ \\ ${ }^{1}$ Theoretical Particle Physics and Cosmology Group, Physics Department, King's College London, \\ University of London, Strand, London WC2R 2LS, United Kingdom \\ ${ }^{2}$ Theoretical Physics Department, CERN, Geneva, Switzerland \\ ${ }^{3}$ School of Physics and Astronomy, University of Minnesota, Minneapolis, Minnesota 55455, USA
}

(Received 12 March 2021; accepted 9 September 2021; published 4 October 2021)

\begin{abstract}
A stochastic gravitational-wave background is expected to emerge from the superposition of numerous gravitational-wave sources of both astrophysical and cosmological origin. A number of cosmological models can have a parity violation, resulting in the generation of circularly polarized gravitational waves. We present a method to search for parity violation in the gravitational-wave data. We first apply this method to the most recent, third, LIGO-Virgo observing run. We then investigate the constraining power of future A + LIGO - Virgo detectors, including KAGRA to the network, for a gravitational-wave background generated by early universe cosmological turbulence.
\end{abstract}

DOI: 10.1103/PhysRevD.104.L081101

\section{INTRODUCTION}

A stochastic gravitational-wave background (SGWB) is generated from the overlap of gravitational waves (GWs) coming from many independent sources. A number of early-universe cosmological sources have been proposed, including GWs sourced from inflation [1], cosmic strings [2], first-order phase transitions (for recent reviews see, e.g., $[3,4])$, or cosmological models inspired from string theory (see, e.g., [5,6]). Searches for an unpolarized isotropic SGWB have been conducted in the past using data gathered by ground-based interferometer detectors LIGO [7] and Virgo [8], and this allowed upper limits on SGWB energy density to be placed [9-11].

A multitude of mechanisms in the early universe can create parity violation [12] that may manifest itself in the production of asymmetric amounts of right- and lefthanded circularly polarized isotropic GWs. Since astrophysical sources of the SGWB are unlikely to have this circular polarization, a detection of such a signal can allow cosmologically sourced GWs to be distinguished from the astrophysically sourced component of the SGWB. A closer analysis of polarized SGWB can place constraints on parity-violating theories.

Numerous parity-violating effects on the SGWB have been studied in the literature, including those resulting from the Chern-Simons gravitational term [13-15] and axion

Published by the American Physical Society under the terms of the Creative Commons Attribution 4.0 International license. Further distribution of this work must maintain attribution to the author(s) and the published article's title, journal citation, and DOI. inflation [16]. Another potential chiral source for early universe SGWB is turbulence in the primordial plasma induced either from cosmological first-order (electroweak or QCD) phase transitions [17-19], or from the primordial magnetic fields that are coupled to the cosmological plasma [20-24]. Parity-violating effects on the SGWB have been explored in detail before [25] from a previous LIGO-Virgo observing run [26], as well as studied in the context of current and future detector capabilities [27-31].

Since turbulence is a stochastic process, the GWs produced in the process are stochastic as well. Similarly, a parity-violating turbulent source will produce circularly polarized GWs. Depending on the helicity strength of the source, there are two types of turbulence GW spectra $[32,33]$. Turbulence dominated by energy dissipation at small scales leads to a helical Kolmogorov (HK) spectrum, whereas turbulence dominated by helicity dissipation at small scales leads to a helicity transfer (HT) spectrum. We focus on models that result in a HK spectrum, and consider the polarization degree associated with them.

In what follows we adopt the formalism of [34] and present a method to detect parity violation in GW data. We first analyse recent data from the third Advanced LIGOVirgo observing (O3) run [10] to place upper limits for a simple power law parity violation model for the normalized GW energy density $\Omega_{\mathrm{GW}}$. We consequently study the SGWB produced by turbulence in the primordial plasma and investigate what upper limits can be placed with the inclusion of KAGRA [35] and improved LIGO-Virgo sensitivities.

The rest of the paper is organized as follows: in Sec. II we present our methodology which we then apply to some parity violation models described in Sec. III. In Sec. IV we 
state our results from existing GW data as well as future prospects, and in Sec. V we discuss our conclusions.

\section{METHOD}

We use the plane-wave expansion of the metric $h_{a b}$ at cosmic time $t$ and position vector $\vec{x}$ [36]:

$h_{a b}(t, \vec{x})=\sum_{A} \int_{-\infty}^{\infty} d f \int_{S^{2}} d \hat{\Omega} h_{A}(f, \hat{\Omega}) e^{-2 \pi i f(t-\vec{x} \cdot \hat{\Omega})} e_{a b}^{A}(\hat{\Omega})$,

where $f$ is the frequency and $e_{a b}^{A}(\hat{\Omega})$ is the polarization tensor for a wave traveling in direction $\hat{\Omega}$. We use the circularly polarized bases $e^{R}=\left(e^{+}+i e^{\times}\right) / \sqrt{2}$ and $e^{L}=$ $\left(e^{+}-i e^{\times}\right) / \sqrt{2}$ (with + and $\times$ the plus and cross polarizations, respectively) to obtain the right- and left-handed modes $h_{R}=\left(h_{+}-i h_{\times}\right) / \sqrt{2}$ and $h_{L}=\left(h_{+}+i h_{\times}\right) / \sqrt{2}$, respectively. Right- and left-handed correlators can then be written as

$$
\begin{aligned}
& \left(\begin{array}{c}
\left\langle h_{R}(f, \hat{\Omega}) h_{R}^{*}\left(f^{\prime}, \hat{\Omega}^{\prime}\right)\right\rangle \\
\left\langle h_{L}(f, \hat{\Omega}) h_{L}^{*}\left(f^{\prime}, \hat{\Omega}^{\prime}\right)\right\rangle
\end{array}\right) \\
& \quad=\frac{\delta\left(f-f^{\prime}\right) \delta^{2}\left(\hat{\Omega}-\hat{\Omega}^{\prime}\right)}{4 \pi}\left(\begin{array}{c}
I(f, \hat{\Omega})+V(f, \hat{\Omega}) \\
I(f, \hat{\Omega})-V(f, \hat{\Omega})
\end{array}\right),
\end{aligned}
$$

where $\langle\cdot\rangle$ represents the ensemble average and $I, V$ are the Stokes parameters, with $V$ characterizing the asymmetry between right- and left-handed polarized waves, and $I(\geq$ $|V|)$ the wave's total amplitude. For $V=0$, Eq. (2) would be simply the correlator for unpolarized isotropic SGWB.

We use the standard cross-correlation estimator [36,37]:

$$
\begin{aligned}
\left\langle\hat{C}_{d_{1} d_{2}}\right\rangle & =\int_{-\infty}^{\infty} d f \int_{-\infty}^{\infty} d f^{\prime} \delta_{T}\left(f-f^{\prime}\right)\left\langle s_{d_{1}}^{*}(f) s_{d_{2}}\left(f^{\prime}\right)\right\rangle \tilde{Q}\left(f^{\prime}\right) \\
& =\frac{3 H_{0}^{2} T}{10 \pi^{2}} \int_{0}^{\infty} d f \frac{\Omega_{\mathrm{GW}}^{\prime}(f) \gamma_{I}^{d_{1} d_{2}}(f) \tilde{Q}(f)}{f^{3}}
\end{aligned}
$$

where

$$
\begin{aligned}
\Omega_{\mathrm{GW}}^{\prime} & =\Omega_{\mathrm{GW}}\left[1+\Pi(f) \frac{\gamma_{V}^{d_{1} d_{2}}(f)}{\gamma_{I}^{d_{1} d_{2}}(f)}\right], \\
\gamma_{I}^{d_{1} d_{2}}(f) & =\frac{5}{8 \pi} \int d \hat{\Omega}\left(F_{d_{1}}^{+} F_{d_{2}}^{+*}+F_{d_{1}}^{\times} F_{d_{2}}^{\times *}\right) e^{2 \pi i f \hat{\Omega} \cdot \Delta \vec{x}}, \\
\gamma_{V}^{d_{1} d_{2}}(f) & =-\frac{5}{8 \pi} \int d \hat{\Omega}\left(F_{d_{1}}^{+} F_{d_{2}}^{\times *}-F_{d_{1}}^{\times} F_{d_{2}}^{+*}\right) e^{2 \pi i f \hat{\Omega} \cdot \Delta \vec{x}},
\end{aligned}
$$

with $H_{0}$ the Hubble parameter, $T$ the observing time, $\delta_{T}(f)=\sin (\pi f T) /(\pi f), \quad \tilde{s}_{d_{1}}(f)$ and $\tilde{s}_{d_{2}}(f)$ the Fourier transforms of the strain time series of two GW detectors (denoted by $\left.d_{1}, d_{2}\right)$. We apply the usual $\tilde{Q}(f)$ as the optimal filter taking into account detectors' strain power spectral densities [37], and $F_{n}^{A}=e_{a b}^{A} d_{n}^{a b}$ stands for the contraction of the tensor modes of polarization $A$ to the $n$th detector's geometry. We denote by $\gamma_{I}^{d_{1} d_{2}}$ the standard overlap reduction function of two detectors $d_{1}, d_{2}$, and by $\gamma_{V}^{d_{1} d_{2}}$ the overlap function associated with the parity violation term. The polarization degree, $\Pi(f)=V(f) / I(f)$, takes on values between -1 (fully left polarization) and 1 (fully right polarization), with $\Pi=0$ being an unpolarized isotropic SGWB.

The variance associated with the estimator $\hat{C}_{d_{1} d_{2}}$ is [36]

$$
\sigma_{d_{1} d_{2}}^{2}=\frac{T}{2} \int_{0}^{\infty} d f P_{d_{1}}(f) P_{d_{2}}(f)|\tilde{Q}(f)|^{2},
$$

where $P_{d_{1}, d_{2}}(f)$ are the one-sided noise power spectral densities of GW detectors $d_{1}, d_{2}$. With each observing run, advancements of noise mitigation methods lower the $P_{d_{i}}(f)$ curves and will eventually uncover the SGWB.

To proceed we perform parameter estimation and fit GW models to data using a hybrid frequentist-Bayesian approach [38]. We construct a Gaussian log-likelihood for a network of $N$ detectors

$$
\log p(\hat{C}(f) \mid \boldsymbol{\theta}) \propto \sum_{i, j>i}^{N} \sum_{f} \frac{\left[\hat{C}_{d_{i} d_{j}}(f)-\Omega_{\mathrm{GW}}^{\prime}(f, \boldsymbol{\theta})\right]^{2}}{\sigma_{d_{i} d_{j}}^{2}(f)},
$$

where $\hat{C}_{d_{i} d_{j}}(f)$ is the frequency-dependent cross-correlation estimator of the SGWB calculated using data from detectors $d_{i}, d_{j}$, and $\sigma_{d_{i} d_{j}}^{2}(f)$ is its variance [37]. ${ }^{1}$ We assume that correlated-noise sources have been either filtered out [39] or accounted for [40]. The normalized GW energy density model we fit to the data is $\Omega_{\mathrm{GW}}^{\prime}(f, \boldsymbol{\theta})$, with parameters $\boldsymbol{\theta}$ including both GW parameters as well as parameters of the $\Pi(f)$ model.

\section{MODELS}

\section{A. SGWB models}

We will apply our method to two classes of GW models. First, we perform a generic search for a parity violating SGWB, with a power-law behavior

$$
\Omega_{\mathrm{GW}}(f)=\Omega_{\mathrm{ref}}\left(f / f_{\text {ref }}\right)^{\alpha},
$$

setting $f_{\text {ref }}=25 \mathrm{~Hz}$. The amplitude prior we use is loguniform between $10^{-13}$ and $10^{-5}$, and the spectral index prior is a Gaussian distribution centred at 0 with a standard deviation 3.5, following the priors used in [10]. We will

\footnotetext{
${ }^{1}$ Searches for the SGWB only use cross-correlation data opposed to autocorrelation as the detector noise is not fully understood, particularly at lower frequencies.
} 
search for this model in the currently available SGWB data and place upper limits on its parameters.

Second, we use a broken power-law spectral shape, motivated by high energy physics. Since we have not detected a SGWB yet, we will investigate detection prospects of this more complicated turbulence model with future improved detector sensitivities and forecast what one can learn about its parameters. Extensions of the Standard Model of particle physics can imply parity violation at the electroweak energy scale being manifested through helical (or chiral) turbulent motion [41,42]. Parity-violating turbulent sources will produce circularly polarized GWs [43], with a broken power-law spectral shape peaking at the characteristic frequency of the source. Recent numerical simulations show that at frequencies below the characteristic frequency $\Omega_{\mathrm{GW}} \sim f$ [44]. Above the characteristic frequency, the decaying power-law depends on the turbulence model [45]. As an example, in our analysis we focus on choice model [46]:

$$
\Omega_{\mathrm{GW}}(f)= \begin{cases}\Omega_{\text {peak }}\left(f / f_{\text {peak }}\right) & , f \leq f_{\text {peak }} \\ \Omega_{\text {peak }}\left(f / f_{\text {peak }}\right)^{-8 / 3} & , f>f_{\text {peak }} .\end{cases}
$$

The peak frequency of the spectrum, $f_{\text {peak }}$, is directly related to the temperature, $T_{*}$, at which the first order phase transition occurred. At the electroweak scale, $T_{*}=100 \mathrm{GeV}$, we expect the GW spectrum to peak in the $\mathrm{mHz}$ range, which has given rise to many LISA-focused turbulence studies. For a higher energy scale $T_{*} \sim 10^{8} \mathrm{GeV}$, one would see a chiral turbulence spectrum that peaks in the LIGO/Virgo range. It is these early universe signatures we search for with the currently operating terrestrial $\mathrm{GW}$ detectors, and therefore we set $f_{\text {peak }}=25 \mathrm{~Hz}$, the most sensitive frequency band. Such a choice of peak frequency will give the most optimistic prospects of detecting a broken power-law SGWB model.

\section{B. Parity violation models}

In addition to modeling the GW spectrum, we need a model for the amount of polarization of the turbulence spectrum. Previous studies [43,47] calculated numerically the net circular polarization of GWs for different initial turbulent conditions to get the polarization degree $\Pi$ over wave number $k$, and found frequency-dependent models of $\Pi$. In the following, we will study both the simplified $\Pi=$ const. model, as well as a frequency-dependent polarization model. In the former simplified case, the prior for $\Pi$ is uniform between -1 and 1 . For the latter case, we adopt the functional form $\Pi(f)= \pm(f / 1 \mathrm{~Hz})^{\beta}$ with a uniform prior on $\beta$ between -2 and 0 . This simple functional form is motivated by the theoretical models predicting $\Pi$ to decay with frequency $[47,48]$. Furthermore, since we consider frequencies larger than $1 \mathrm{~Hz}$ (terrestrial detectors are limited by seismic noise at low frequencies), this choice

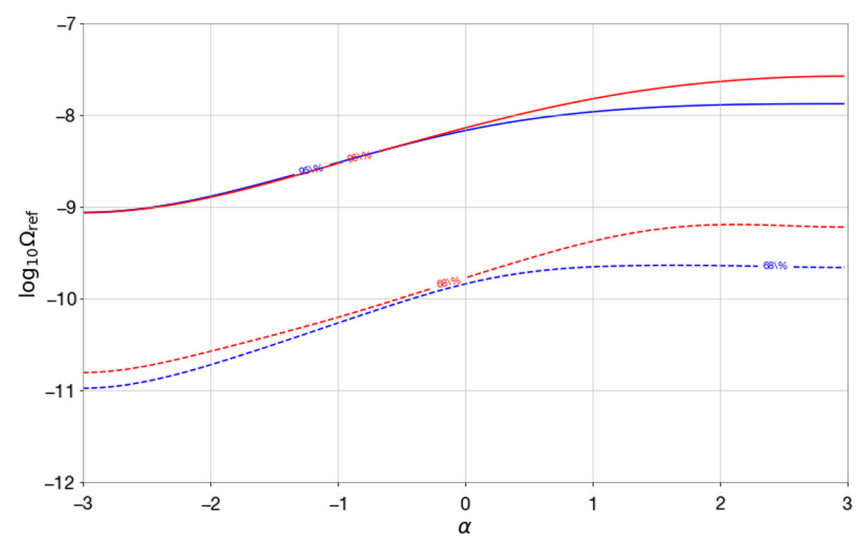

FIG. 1. $\Omega_{\text {ref }}-\alpha$ confidence curve at $95 \%$ (solid) and $68 \%$ (dashed) level for assumed $\Pi= \pm 1$. We see more stringent constraints in the $\Pi=1$ case (blue) than with the $\Pi=-1$ case (red).

ensures that $\Pi(f)$ is well defined and remains within the physical range $[-1,1]$.

\section{RESULTS}

We place upper limits on parameters of the simpler, power-law, SGWB model using data from the recent Advanced LIGO-Virgo O3 observing run. Following this, we discuss detection prospects of model-dependent, broken power-law turbulence spectra as the sensitivity of the interferometers increases and more interferometers are added to the network.

\section{A. $\mathrm{O3}$ results}

We search for a power-law SGWB spectrum, as described in Eq. (7), with a simple $\Pi=$ const. polarization model in the recent one year observation $\mathrm{O} 3$ data [11]. The data consists of cross-correlated strain series from Hanford $(\mathrm{H})$, Livingston (L) and Virgo (V) detectors, and represents the first time Virgo has been included at Advanced sensitivity. We find no preference for a particular $\Pi$ value in the $[-1,1]$ prior range. We find the upper limit on the amplitude of the power law to be $\Omega_{\mathrm{ref}}^{95 \%}=7.0 \times 10^{-9}$. Calculating the Bayes factor, we find $\ln \mathcal{B}_{\Pi=0}^{\Pi \neq 0}=-0.02$, concluding that there is no preference for parity violation models versus no parity violation ones.

It is worth noting that we found the HLV network to be more sensitive to right-hand polarizations compared to lefthand ones. Plotting the $\Omega_{\text {ref }}$ versus $\alpha$ confidence curve generated from $\mathrm{O} 3$ data, one observes that it is easier to constrain $\Omega_{\text {ref }}$ for entirely right-handed polarized GWs $(\Pi=1)$ than it is for left-handed $(\Pi=-1)$ ones, see Fig. 1. Excluding the HL detector baseline however, results to a less obvious polarization bias for right- or lefthanded GWs.

To understand the origin of this bias we investigate the asymmetry in the overlap reduction ratio, $\varsigma^{\mathrm{HL}} \equiv \gamma_{V}^{\mathrm{HL}} / \gamma_{I}^{\mathrm{HL}}$, 

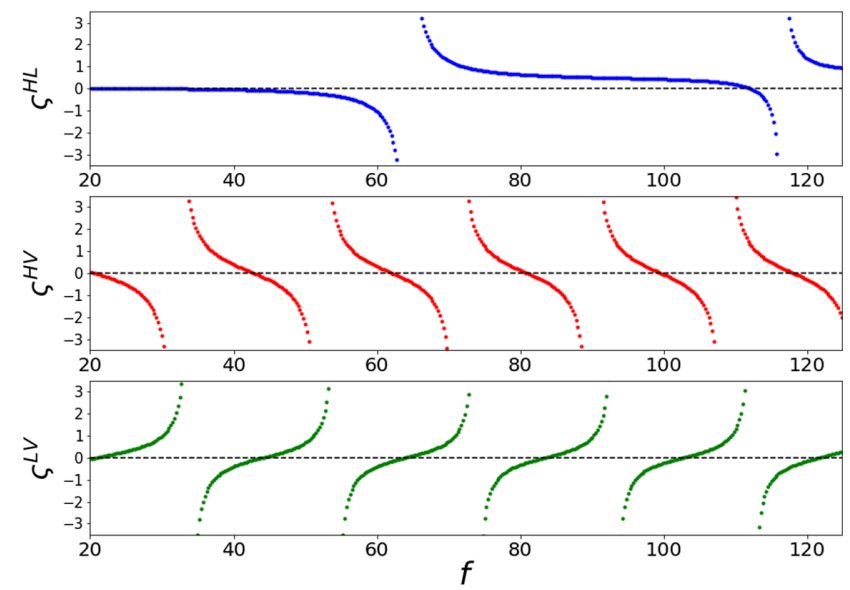

FIG. 2. Overlap reduction function ratio $\varsigma$ for HL (top), HV (middle), and LV (bottom) baselines.

for the HL detector pairing plotted in Fig. 2, along with the corresponding overlap reduction ratios $\varsigma^{\mathrm{HV}}$ and $\varsigma^{\mathrm{LV}}$ for the $\mathrm{HV}$ and $\mathrm{LV}$ baselines, respectively. While $\varsigma^{\mathrm{HV}}$ and $\varsigma^{\mathrm{LV}}$ are roughly periodic in the considered frequency range $(\mathrm{f} \lesssim 130 \mathrm{~Hz}), \varsigma^{\mathrm{HL}}$ is preferentially positive in this frequency range. Preferentially positive $\varsigma^{\mathrm{HL}}$ combined with $\Pi>0$ in Eq. (4) leads to a larger $\Omega_{\mathrm{GW}}^{\prime}$, hence leading to stricter constraints on right-hand polarized signals.

In addition to the frequency-independent, $\Pi(f)=$ const. model, we search for a power-law parity violation signal, assuming a right- and left-handed frequency-dependent degree of polarization $\Pi(f)= \pm(f / 1 \mathrm{~Hz})^{\beta}$. There is no detection of a GW power-law signal with frequencydependent polarization degree, but for an assumed rightand left-handed polarization we can place an upper limit on the amplitude, $\Omega_{\mathrm{ref}}^{95 \%}=4.9 \times 10^{-9}$ and $\Omega_{\mathrm{ref}}^{95 \%}=7.4 \times 10^{-9}$, respectively, after marginalizing over $\alpha$ and $\beta$. Positive polarization was expected to have stricter upper bounds based on HLV network's higher sensitivity to entirely righthand polarization seen in Fig. 1. Since more stringent, we only present positive polarization results. Figure 3 shows posterior distribution of amplitude, $\Omega_{\mathrm{GW}}$, and spectral index, $\alpha$, of a power-law GW signal, as well as the posterior of the polarization degree parameter, $\beta$. The $\alpha$ posterior is similar to the Gaussian prior distribution, implying that we cannot deduce anything about the spectral index, $\alpha$, of the $\mathrm{GW}$ power law from the $\mathrm{O} 3$ data. Finally, with $\mathrm{O} 3$ data, we do not constrain the $\beta$ parameter space.

Since we see no detection of a parity-violating signal in O3 data when we use a simple power-law model with a frequency-independent polarization degree, as well as a frequency-dependent one, we do not proceed to search for the broken power law model in Eq. (8) - such a model would also be undetectable with the data from the third observing run. Instead, we study the prospects of detecting the complex model with a more sensitive GW detector network.

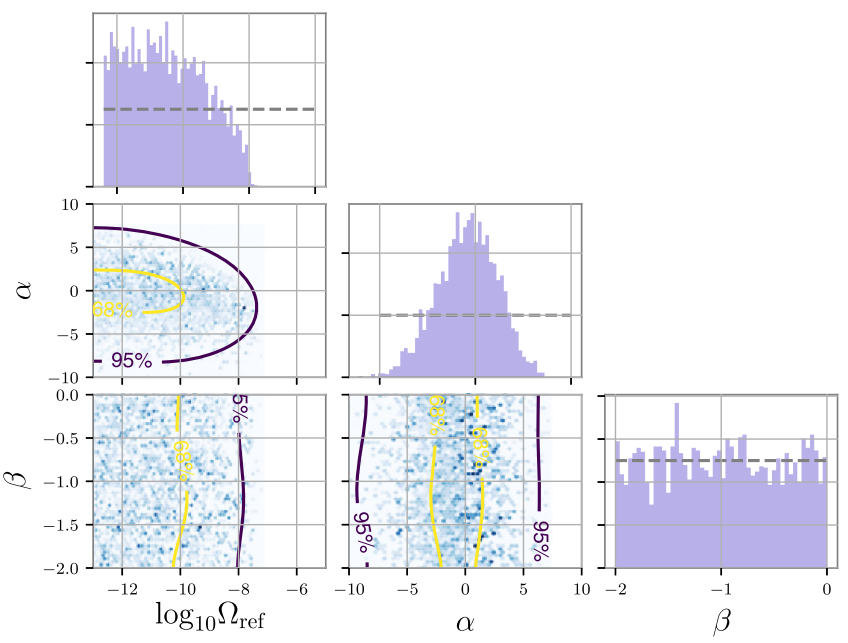

FIG. 3. $95 \%$ confidence limit and posterior distribution obtained using $\mathrm{O} 3$ data for the model of power-law SGWB spectrum with parity violation $\Pi(f)=(f / 1 \mathrm{~Hz})^{\beta}$.

\section{B. Future prospects}

With each observing LIGO-Virgo run, we see improved upper limits on the SGWB, expecting to have a detection in one of the future upgrades of the detector network. Let us therefore investigate the possibility of detecting a parity violation signal with the A + sensitivity of LIGO, $\mathrm{AdV}+$ sensitivity of Virgo, and including KAGRA at Design sensitivity to the network [49]. We simulate the cross-correlation function defined in Eq. (3) that contains a GW signal as well as instrumental noise of the detectors. We note that our simulations are for three years observation time. Adding more GW detectors to the network and extending the observation time both lead to improvements in our sensitivity.

Ultimately, we would like to explore the prospects of a detection of a physically motivated, parity-violating signal. This is why in the following section we focus on the detection of a turbulence signal, and not that of a simple power law model. We simulate a broken power law $\Omega_{\mathrm{GW}}$, induced by turbulence as described in Sec. III [cf. Eq. (8)]. To investigate the detection prospects of such a signal, we vary the amplitude of the simulated spectrum by doing 1000 simulations log-spaced between $\Omega_{\text {peak }} \in\left[10^{-10}, 10^{-7}\right]$. Going forward, we assume the polarization dependence of the simulated spectrum is given by right-handed polarization $\Pi(f)=(f / 1 \mathrm{~Hz})^{-1 / 2}$, as our results indicated stronger positive polarization sensitivity in the HLV network. We discuss below our results and their dependence on a deviation of the polarization parameter $\beta$ from the $-1 / 2$ value.

Figure 4 shows the variation of signal-to-noise Bayes factor, $\mathcal{B}$, of the simulations, focusing particularly in the region $\Omega_{\text {peak }} \in\left[10^{-10}, 10^{-8}\right]$. A $\ln \mathcal{B}$ factor of 8 is equivalent to a frequentist SNR of 4 [36], and as such, we take this value to be our detection threshold. Consequently, all 


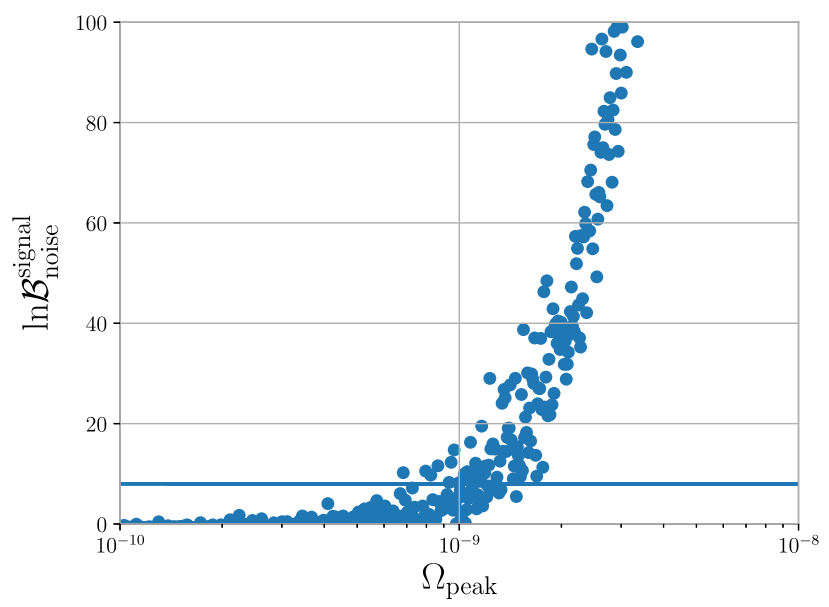

FIG. 4. Bayes factor as a function of amplitude of the simulated signal. Each point represents one of our 1000 simulations. The solid line represents $\ln \mathcal{B}_{\text {noise }}^{\text {signal }}=8$.

points above the solid line in Fig. 4 will be considered as detected. This leads to an upper limit of $\Omega_{\text {peak }}=1.5 \times 10^{-9}$; any louder signal is expected to be detected with great significance by the $\mathrm{A}+$ detectors.

However, even if we can confidently claim a detection of a turbulent, broken power-law SGWB, we might not be able to constrain its polarization. Repeated simulations show that the spectral amplitude $\Omega_{\text {peak }}$ plays the main role in the recovery of the polarization content. More precisely, we find that stronger signals yield better results for the recovery of the $\Pi(f)$ model, i.e. of the $\beta$ parameter. Our analysis also shows that the inclusion of simulated data from the Virgo and KAGRA detectors is critical in recovering the polarization of a simulated SGWB. We find that for simulations with amplitude $\Omega_{\text {peak }} \geq 5 \times 10^{-8}$, we confidently recover the $\beta=-1 / 2$ value, see Fig. 5 . We quantify our confidence in recovery of $\beta$ by requiring $95 \%(2 \sigma)$ of its posterior distribution to be within 0.1 of the simulated value.

Therefore, we conclude that the amplitude of the GW spectrum needs to be more than 30 times larger than its detection threshold in order to recover the $\beta=-1 / 2$ parameter value, and detect a polarization. Only with such a strong detection, one can study the polarization model and its implications for parity violation theories.

Weaker SGWB may still allow us to place an upper limit on $\beta$. Posteriors for these weak SGWB simulations are skewed toward the lower end of the $\beta$ prior. To demonstrate this, Fig. 6 shows the variation of the median value of $\beta$ posteriors as a function of the simulated spectral amplitude. We find that the median of the posterior starts to deviate downward from $\beta=-1 / 2$ for $\Omega_{\text {peak }}<5 \times 10^{-8}$, agreeing with our previously stated definition of confident recovery. This downward skewness is further confirmed by the variations of the first and third quartiles of the posteriors (not shown). For even weaker simulations,

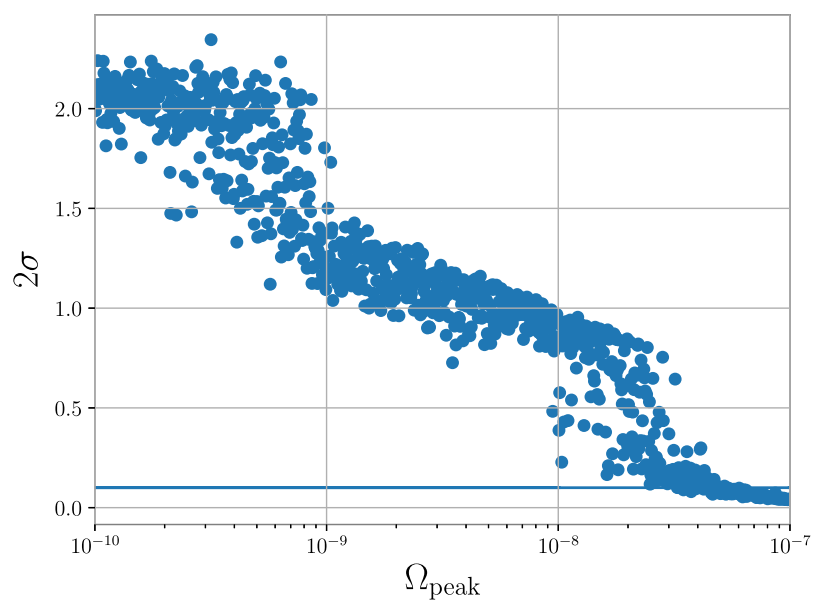

FIG. 5. Variation of $2 \sigma$ value of the $\beta$ posterior for each of the 1000 simulations. The solid line represents $2 \sigma=0.1$.

$\Omega_{\text {peak }} \lesssim 5 \times 10^{-9}$, the signal becomes too weak to constrain polarization at all.

To check the dependence of our results on the simulated value of the $\beta$ parameter, we repeat the analysis for $\beta=-1$ and 0 [47]. The detection threshold for each of the data sets is the same as before, $\Omega_{\text {peak }}=1.5 \times 10^{-9}$, suggesting that when we claim a detection, it will be independent of the amount of polarization of the signal. However, the signal strength needed to successfully recover $\beta$ depends on the polarization model. Namely, the smaller the value of $\beta$ is, the stronger the simulation amplitude is needed. For $\beta=-1$ we are unable to recover it within our simulation range. The $\beta=0$ simulation, with a frequency-independent polarization, is recovered with signals of amplitude $\Omega_{\text {peak }}=1 \times 10^{-8}$, only 7 times stronger than the simulation threshold. The only simulation that successfully recovers $\beta$, and is not already ruled out by the first three LIGO-Virgo observing runs, is $\beta=0$, implying that even if we include 4

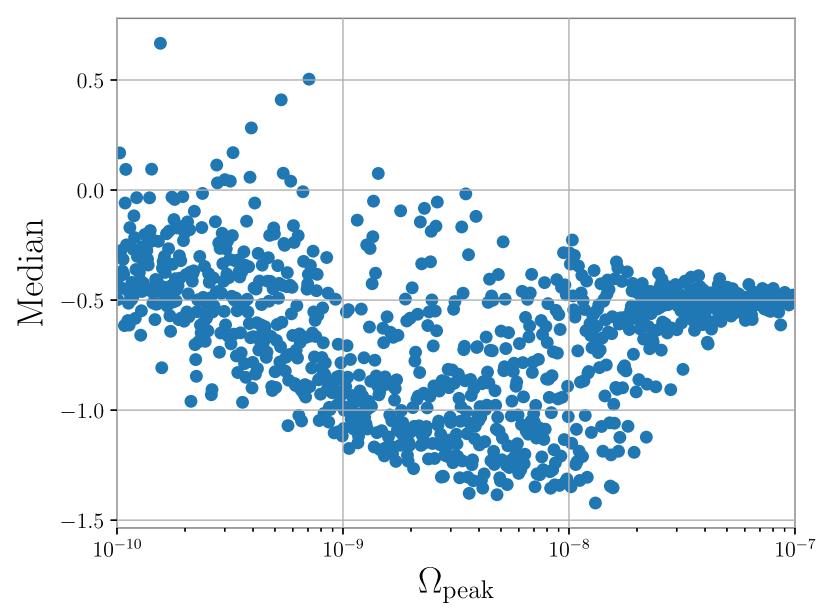

FIG. 6. Variation of the median of the $\beta$ posterior for each of the 1000 simulations. 
detectors, and consider 3 years of observation time, it will be challenging to probe frequency-dependent polarization models.

\section{CONCLUSIONS}

We searched for SGWB generated by parity violation sources in recent $\mathrm{GW}$ data $(\mathrm{O} 3)$ and simulated $\mathrm{GW}$ data (future sensitivities of LIGO/Virgo/KAGRA detectors). We found no evidence for such a signal in $\mathrm{O} 3$ data and we placed an upper limit on the amplitude of a power-law GW model, $\Omega_{\text {ref }}^{95 \%}=4.9 \times 10^{-9}, 7.4 \times 10^{-9}$ for right- and lefthand polarization, respectively. A bias for constraining right-handed polarized waves was found to be due to the geometry of the Hanford-Livingston detector baseline, leading to better constraints of $\Pi>0$ polarizations.

When simulating data for future detection prospects, we considered a chiral turbulence source in the early universe. The results we obtained are model-dependent. For an SGWB modeled as a broken power-law (c.f. Eq. (8), the LIGO-Virgo-KAGRA detector network is sensitive to peak amplitudes down to $\Omega_{\text {peak }}=1.5 \times 10^{-9}$. Our analysis showed that we are able to better estimate the parameters of the parity violation model for stronger simulated GW backgrounds, as well as when the Virgo/KAGRA detectors are included in the analysis, highlighting the importance of having a multi-detector network. For $\beta=-1 / 2$ we found that successful recovery of $\beta$ requires SGWB amplitude of at least $\Omega_{\text {peak }}=5 \times 10^{-8}$, which is excluded by existing GW data. Hence, even if we detect a turbulence signal, we might not be able to deduce its polarization. The recovery of frequency-independent polarization $(\beta=0)$ showed more promising results and we might be able to constrain such signals in the future observing runs.

Although this study used the current LIGO and Virgo detectors as well as the upcoming KAGRA detector, it would be interesting to apply the same study to additional ground detectors added to the network such as LIGO-India, as well as other planned terrestrial detectors (Einstein Telescope [30,50], Cosmic Explorer [51]), or the spaceborne LISA [28-30,52]. Note that due to uncertainty in geographical locations (i.e. uncertainty in overlap reduction functions) of the planned terrestrial detectors, the study cannot be extended to these at the moment.

In our analysis, we focused on chiral turbulence from an early universe phase transition, but there are other sources of parity violation, like the well-studied chiral inflaton field [53]. The method we have presented here could be easily adapted in such a scenario.

Unresolved compact binary coalescences (CBCs) are expected to be the dominant contribution to the SGWB. Hence one could study the SGWB with CBC and parity violation signals being both present. We leave this as a future work.

\section{ACKNOWLEDGMENTS}

It is a pleasure to thank Tina Kahniashvili and Axel Brandenburg on fruitful discussions about turbulence. We acknowledge computational resources provided by the LIGO Laboratory and supported by National Science Foundation Grants No. PHY-0757058 and No. PHY0823459. This paper has been given LIGO DCC number LIGO-P2100076. K. M. is supported by King's College London through a Postgraduate International Scholarship. M. S. is supported in part by the Science and Technology Facility Council (STFC), United Kingdom, under the research Grant No. ST/P000258/1. V. M. is supported by the NSF Grant No. PHY-1806630. Software packages used in this paper are MATPLOTLIB [54], NUMPY [55], BILBY [56], CHAINCONSUMER [57].
[1] Daniel J. H. Chung, Edward W. Kolb, Antonio Riotto, and Igor I. Tkachev, Phys. Rev. D 62, 043508 (2000).

[2] A. Vilenkin and T. Damour, Phys. Rev. Lett. 85, 3761 (2000).

[3] C. Caprini, M. Hindmarsh, T. Konstandin, J. Kozaczuk, G. Nardini, J. M. No, A. Petiteau, P. Schwaller, G. Servant et al., J. Cosmol. Astropart. Phys. 04 (2016) 601.

[4] M. Hindmarsh M. Lüben, J. Lumma, and M. Pauly, SciPost Phys. Lect. Notes. 24, 24 (2020).

[5] M. Gasperini and G. Veneziano, Astropart. Phys. 1, 317 (1993).

[6] M. Gasperini, Elements of String Cosmology (Cambridge University Press, 2007).

[7] J. Aasi, B. P. Abbott, R. Abbott, T. Abbott, M. R. Abernathy, K. Ackley, C. Adams, T. Adams, P. Addesso, R. X. A et al., Classical Quantum Gravity 32, 115012 (2015).
[8] F. Acernese et al. (Virgo Collaboration), Classical Quantum Gravity 32, 024001 (2015).

[9] B. P. Abbott et al., Phys. Rev. D 100, 061101 (2019).

[10] R. Abbott et al., Phys. Rev. D 104, 022004 (2021).

[11] R. Abbott et al., https://dcc.ligo.org/G2001287/public.

[12] S. H. Alexander, M. E. Peskin, and M. M. Sheikh-Jabbari, Phys. Rev. Lett. 96, 081301 (2006).

[13] M. Satoh, S. Kanno, and J. Soda, Phys. Rev. D 77, 023526 (2008).

[14] N. Bartolo, L. Caloni, G. Orlando, and A. Ricciardone, J. Cosmol. Astropart. Phys. 03 (2021) 073.

[15] T. Takahashi and J. Soda, Phys. Rev. Lett. 102, 231301 (2009).

[16] N. Barnaby and M. Peloso, Phys. Rev. Lett. 106, 181301 (2011). 
[17] M. Kamionkowski, A. Kosowsky, and M. S. Turner, Phys. Rev. D 49, 2837 (1994).

[18] E. Witten, Phys. Rev. D 30, 272 (1984).

[19] C. J. Hogan, Mon. Not. R. Astron. Soc. 218, 629 (1986).

[20] A. Brandenburg, K. Enqvist, and P. Olesen, Phys. Rev. D 54, 1291 (1996).

[21] M. Christensson, M. Hindmarsh, and A. Brandenburg, Phys. Rev. E 64, 056405 (2001).

[22] T. Kahniashvili, A. Brandenburg, A. G. Tevzadze, and B. Ratra, Phys. Rev. D 81, 123002 (2010).

[23] A. Brandenburg, T. Kahniashvili, S. Mandal, A. R. Pol, A. G. Tevzadze, and T. Vachaspati, Phys. Rev. Fluids 4 (2019).

[24] A. Brandenburg, Y. He, T. Kahniashvili, M. Rheinhardt, and J. Schober, Astrophys. J. 911, 110 (2021).

[25] S. G. Crowder, R. Namba, V. Mandic, S. Mukohyama, and M. Peloso, Phys. Lett. B 726, 66 (2013).

[26] The LIGO Scientific and The Virgo Collaborations, Nature (London) 460, 990 (2009).

[27] N. Seto, Phys. Rev. D 75, 061302 (2007).

[28] A. Ricciardone, J. Phys. Conf. Ser. 840, 012030 (2017).

[29] G. Orlando, M. Pieroni, and A. Ricciardone, J. Cosmol. Astropart. Phys. 03 (2021) 069.

[30] V. Domcke, J. García-Bellido, M. Peloso M. Pieroni A. Ricciardone L. Sorbo, and G. Tasinato, J. Cosmol. Astropart. Phys. 05 (2020) 028.

[31] P. Xu, Z. Wang, and L. Qiang, Phys. Lett. B 789, 378 (2019).

[32] S. S. Moiseev and O. Chkhetiani, J. Exp. Theor. Phys. 83, 192 (1996).

[33] M. Lesieur (1997).

[34] N. Seto and A. Taruya, Phys. Rev. Lett. 99, 121101 (2007).

[35] T. Akutsu et al., arXiv:2005.05574.

[36] N. J. Cornish and J. D. Romano, Living Rev. Relativity 20, 2 (2017).
[37] B. Allen and J. D. Romano, Phys. Rev. D 59, 102001 (1999).

[38] A. Matas and J. D. Romano, Phys. Rev. D 103, 062003 (2021).

[39] M. W Coughlin et al., Phys. Rev. D 97, 102007 (2018).

[40] P. M. Meyers, K. Martinovic, N. Christensen, and M. Sakellariadou, Phys. Rev. D 102, 102005 (2020).

[41] A. J. Long, E. Sabancilar, and T. Vachaspati, J. Cosmol. Astropart. Phys. 02 (2014) 036.

[42] G. C. Dorsch, S. J. Huber, T. Konstandin, and J. M. No, J. Cosmol. Astropart. Phys. 05 (2017) 052.

[43] T. Kahniashvili, arXiv:astro-ph/0508459.

[44] A. R. Pol, S. Mandal, A. Brandenburg, T. Kahniashvili, and A. Kosowsky, Phys. Rev. D 102, 083512 (2020).

[45] D. J. Weir, Phil. Trans. R. Soc. A 376, 20170126 (2018).

[46] A. Kosowsky, A. Mack, and T. Kahniashvili, Phys. Rev. D 66, 102005 (2002).

[47] T. Kahniashvili, A. Brandenburg, G. Gogoberidze, S. Mandal, and A. Roper Pol, Phys. Rev. Research 3, 013193 (2021).

[48] L. Kisslinger and T. Kahniashvili, Phys. Rev. D 92, 043006 (2015).

[49] B. P. Abbott et al., Living Rev. Relativity 21, 3 (2018).

[50] M. Punturo et al., Classical Quantum Gravity 27, 084007 (2010).

[51] D. Reitze et al., Bull. Am. Astron. Soc. 51, 035 (2019).

[52] P. Amaro-Seoane et al., arXiv:1702.00786.

[53] L. Sorbo, J. Cosmol. Astropart. Phys. 06 (2011) 003.

[54] J. D. Hunter, Comput. Sci. Eng. 9, 90 (2007).

[55] S. van der Walt, S. C. Colbert, and G. Varoquaux, Comput. Sci. Eng. 13, 22 (2011).

[56] G. Ashton et al., Astrophys. J. Suppl. Ser. 241, 27 (2019).

[57] S. R. Hinton, J. Open Source Softw. 1, 00045 (2016). 